\title{
Numerical study of blood fluid rheology in the abdominal aorta
}

\author{
F. Carneiro ${ }^{1}$, V. Gama Ribeiro ${ }^{2}$, J. C. F. Teixeira ${ }^{1}$ \\ \& S. F. C. F. Teixeira ${ }^{3}$ \\ ${ }^{1}$ Universidade do Minho, Departamento de Engenharia Mecânica, \\ Campus de Azurém, 4800-058 Guimarães, Portugal \\ ${ }^{2}$ Centro Hospitalar de Vila Nova de Gaia, \\ Rua Conceição Fernandes - Vilar de Andorinho \\ 4430-502 Vila Nova de Gaia, Portugal \\ ${ }^{3}$ Universidade do Minho, Departamento de Produção e Sistemas, \\ Campus de Azurém, 4800-058 Guimarães, Portugal
}

\begin{abstract}
The present paper reports the numerical modelling of blood flow in the abdominal aorta. The effect of blood rheology on flow dynamics was studied in a 3D model of the abdominal aorta bifurcation into the iliac arteries using the FLUENT software. Newtonian and non-Newtonian models for the fluid viscosity have been implemented for assessing the size of the recirculation zone; velocity profile and wall shear stress distribution. A recirculation zone and low wall shear stress were observed for both cases in the vicinity of the iliac bifurcation. These are believed to play an important role in the atherosclerosis lesions. Both Newtonian and non-Newtonian simulations in the vicinity of the iliac bifurcation presented minor differences. This study provides a better understanding of atherosclerosis development in the iliac arteries bifurcation, which may improve the design of stent devices.
\end{abstract}

Keywords: blood rheology, cardiovascular modelling, non-Newtonian.

\section{Introduction}

Atherosclerosis is a very common disease, characterized by the deposition of fatty material in the internal walls of blood arteries, causing their thickening and 
reduction of the available artery cross section. It is often associated with abnormal flow, happening mostly in regions of flow separation, recirculationstagnation zones, turbulence and low wall shear stress, which are believed to be atheromatic factors [1]. The blood flow behaviour, or hemodynamics, is dependent on different factors, such as the artery geometry and blood rheology.

The blood is a complex medium, composed of plasma, a Newtonian fluid, and a suspension of various cells, such as erythrocytes, leucocytes and platelets. The blood cells are about $45 \%$ blood by volume, forming a non-Newtonian fluid, with shear-thinning viscoelastic behaviour. These blood cells are related to its microscopic structures, such as aggregation, deformation and alignment of the erythrocytes [2]. Owens [3] developed a new microstructure-based constitutive model for blood that exhibits shear-thinning and viscoelasticity. However, in most CFD (Computational Fluid Dynamics) simulations, blood is modelled as a Newtonian fluid, particularly in large arteries, where the influence of shearthinning properties is not significant. The viscoelastic properties of blood are also often ignored.

Johnston et al. [4] concluded that the Newtonian model of blood viscosity in coronary arteries is an approximation just as good in regions of mid-range to high shear. Non-Newtonian effects were investigated by some authors in carotid arteries [5,6]. Perktold and Rappitsch [5] concluded that the Casson model does not describe well the viscosity in pulsatile flow. The experimental study of Gijsen et al. [6] proves that the blood flow should be simulated by the non-Newtonian viscous model. Gijsen et al. [6] studied the Carreau-Yasuda model, which is still being used by Abraham and Chen. This model describes the blood flow behaviour at both low and high shear stress. They suggest that the non-Newtonian properties of blood are an important feature in the hemodynamics, which affect the interaction between the fluid and the vessel. This interaction plays an important role in vascular biology and pathophysiology.

This paper reports the influence of fluid properties on the incompressible steady state blood flow near the abdominal aorta bifurcation into the two iliac arteries. The three dimensional model is simulated with the CFD Fluent software, assuming arteries as rigid walls and turbulence of the blood flow. The results presented in this paper discuss the influence of the blood rheology upon the wall shear stress values in the vicinity of the aorta bifurcation and the size of recirculation zones.

This work is a part of a wider effort to couple experimental data with numerical simulations for the knowledge improvement of the hemodynamic in arteries bifurcations.

\section{Model and numerical solution}

\subsection{Geometry model}

A schematic representation of the three-dimensional geometry, considered in this investigation, and the relevant details are given in figure 1. 


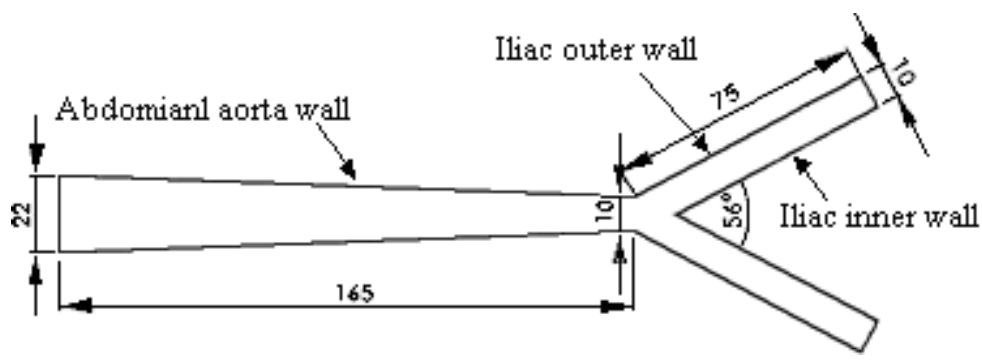

Figure 1: Geometry of the abdominal aorta in the iliac arteries.

The abdominal aorta bifurcation of the iliac arteries is modelled as a rigid wall with a circular cross section. This approach enables the subsequent model validation with experimental data.

\subsection{Mesh generation}

The geometry and the computational mesh were created using the GAMBIT software. The number of control volumes is a balance between the desired low computational time and a high precision. To reduce the computational time, a symmetrical geometry is assumed in the planes $\mathrm{y}=0$ and $\mathrm{z}=0$ (Figure 2), reducing the domain to just one quarter. The domain was decomposed into four connected subdomains. Specifically, two of these are at the bifurcation region, to refine the local mesh, where higher velocity gradients are expected. The model was meshed with hexahedral elements of different size: $2.3 \mathrm{~mm}$ for the aorta, $0.75 \mathrm{~mm}$ in the bifurcation zone and $1.4 \mathrm{~mm}$ for the iliac. Boundary layers were considered at the cross section throughout the entire domain, to refine the mesh near the walls, and at the inlet and outlet faces of abdominal aorta (Table 1). The resulting computational domain for this model was built with 13376 hexahedral elements (Figure 2).

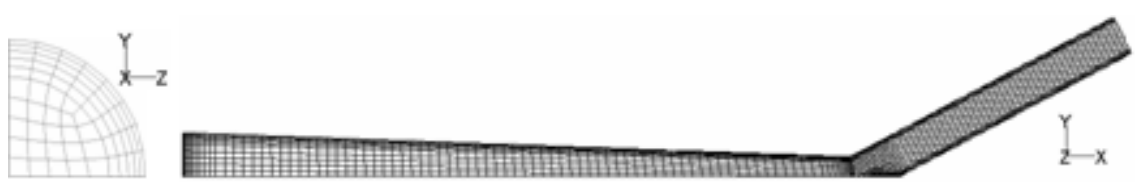

Figure 2: $\quad$ Computational mesh the model (inlet and $z=0$ planes).

Mesh independence has been confirmed by increasing the number of elements. Another mesh was developed with refinements in axial and radial directions, yielding a total of 34960 elements [7]. The differences between both meshes regarding the length of the recirculation zone are within $0.9 \%$.

The domain discretization into control volumes is necessary for the finite volume method used in the FLUENT. This CFD software solves the equations of mass, momentum and the turbulence in each control volume, guarantying conservation for each variable. 
Table 1: $\quad$ Properties of the grid refinements: first element size $(\mathrm{mm})$ and number of elements.

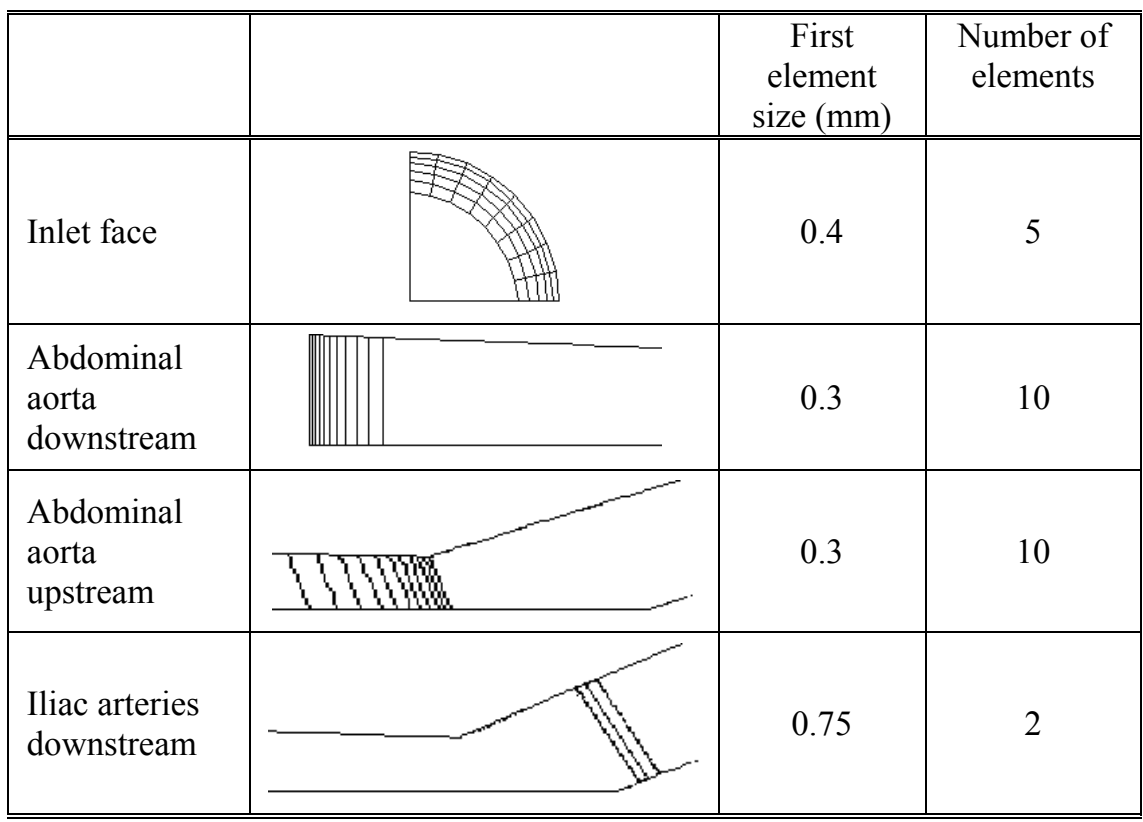

\subsection{Numerical procedure}

Blood was modelled as an incompressible fluid, with the density of $1057 \mathrm{~kg} / \mathrm{m}^{3}$. The uniform inlet velocity is $0.234 \mathrm{~m} / \mathrm{s}$. The inflow is laminar and the generated turbulence was simulated by the $\mathrm{k}-\varepsilon$ model with enhanced wall treatment. The three-dimensional conservation equations for mass (equation 1) and momentum (equation 2) are:

$$
\begin{gathered}
\nabla \vec{v}=0 \\
\frac{\partial}{\partial t}(\rho \vec{v})+\nabla \cdot(\rho \vec{v} \vec{v})=-\nabla p+\nabla \cdot(\overline{\bar{\tau}})
\end{gathered}
$$

where $\vec{v}$ is the fluid velocity vector, $\rho$ is the density, $p$ is the static pressure and $\overline{\bar{\tau}}$ is the stress tensor.

The rheological model for blood viscosity is a key factor in the simulation of hemodynamics flows. To study the influence of blood rheology on the atherosclerosis development the flow was modelled either as a Newtonian fluid or as a non-Newtonian fluid described by the Carreau model.

For the Newtonian option the dynamic viscosity is constant $\left(0.0035 \mathrm{~kg} \cdot \mathrm{m}^{-1} \mathrm{~s}^{-1}\right)$ and the shear stress proportional to the rate-of-deformation tensor $\overline{\bar{D}}$ as described by the following equation:

$$
\overline{\bar{\tau}}=\mu \overline{\bar{D}}
$$


where $\mu$ is the dynamic viscosity for a Newtonian fluid and $\overline{\bar{D}}$ is defined by

$$
\overline{\bar{D}}=\left(\frac{\partial u_{j}}{\partial x_{i}}+\frac{\partial u_{i}}{\partial x_{j}}\right)
$$

For the non-Newtonian fluid the shear viscosity $(\eta)$ is a function of the shear rate $(\dot{\gamma})$ and the shear stress can be similarly written as:

with

$$
\overline{\bar{\tau}}=\eta(\overline{\bar{D}}) \overline{\bar{D}}
$$

$$
\dot{\gamma}=\sqrt{\frac{1}{2} \overline{\bar{D}}: \overline{\bar{D}}}
$$

The non-Newtonian Carreau shear-thinning model used is:

$$
\eta=\eta_{\infty}+\left(\eta_{0}-\eta_{\infty}\right)\left[1+(\dot{\gamma} \lambda)^{2}\right]^{(n-1) / 2}
$$

where $\eta$ is the viscosity $\left(\mathrm{kg} \cdot \mathrm{m}^{-1} \mathrm{~s}^{-1}\right), \eta_{0}$ and $\eta_{\infty}$ are, respectively, the upper and lower limiting values of the fluid viscosity, $n$ is the power-law index, $\lambda$ is the time constant, and $\dot{\gamma}$ the shear rate. In this work, the Carreau model parameters are: $\eta_{0}=22 \times 10^{-3}, \eta_{\infty}=2.2 \times 10^{-3}, n=0.392$ and $\lambda=0.110$.

In the finite volume method the partial differential equations are approximated by a set of algebraic equations over the computational domain that is subsequently solved. Solutions are obtained iteratively using the segregated solver with the SIMPLE algorithm, and the convergence is accepted when the residuals are below 1e-05. It was used the standard scheme to solve pressure equation and the second order upwind scheme to solve momentum, turbulence kinetic energy and turbulence dissipation rate equations.

\section{Results}

The equations and the numerical procedure described above were applied to both Newtonian and non-Newtonian fluids through the abdominal aorta bifurcation into the iliac arteries. The velocity distribution, in the vicinity of the bifurcation, is shown in figure 3.

The velocity distributions, for Newtonian and non-Newtonian solutions, are very similar, particularly near the axis. The velocity vectors at plane $z=0$, in the vicinity of the bifurcation, are shown in Figure 4, for the non-Newtonian fluid.

A recirculation zone is developed downstream the bifurcation with separation of flow. Velocity profile just upstream the bifurcation, at $165 \mathrm{~mm}$, can be observed in detail in the Figure 4.

Although the results are very similar, the higher velocity observed for the Newtonian fluid (1.7\% higher) yields a lower velocity gradient at the wall, when compared with the non-Newtonian fluid. Such differences are shown in Table 2, which includes the velocity and the wall shear stress (WSS) peak and the recirculation size of recirculation zone near the wall, for the Newtonian and non- 

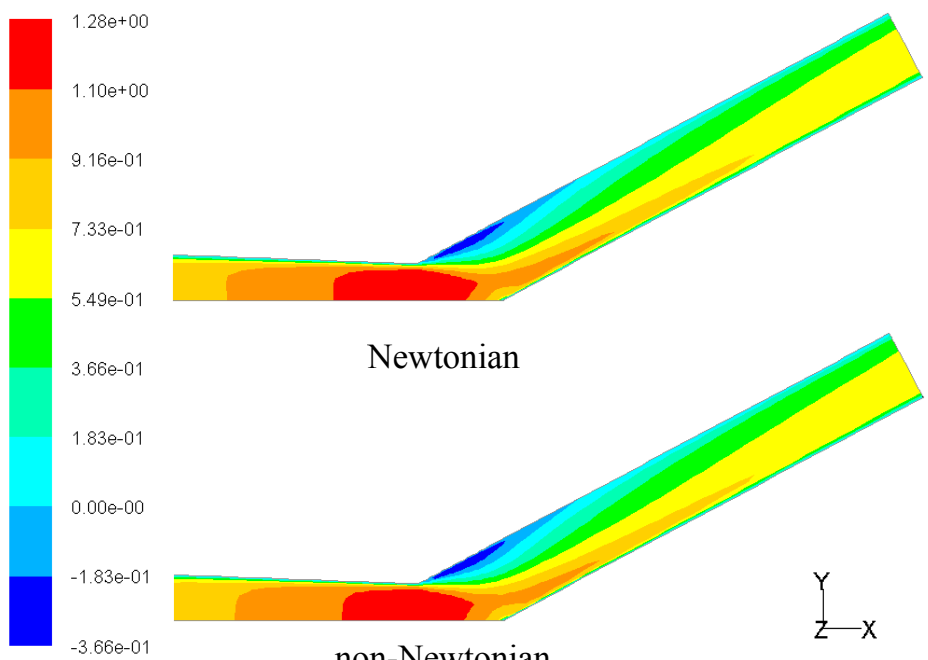

Figure 3: Velocity contours for the Newtonian and non-Newtonian fluids, at plane $z=0$.

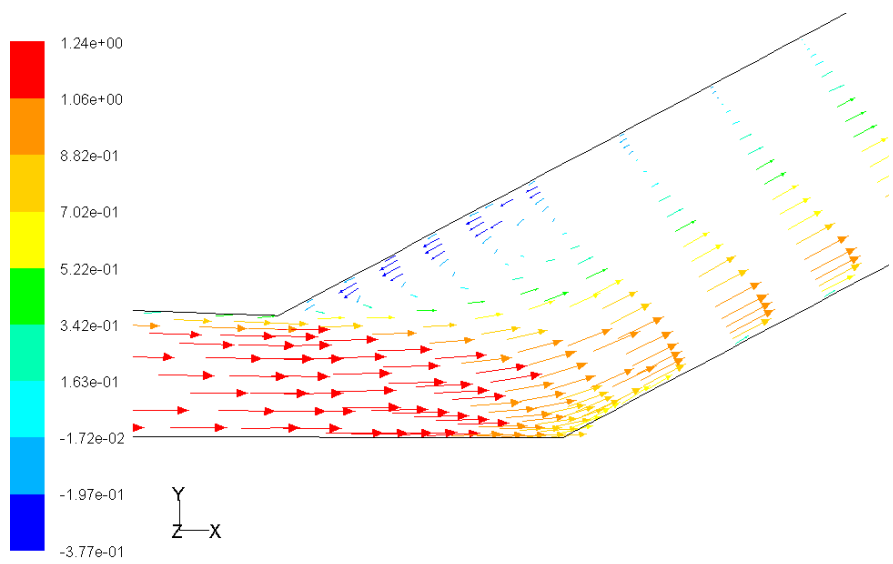

Figure 4: $\quad$ Velocity vectors for the non-Newtonian fluid.

Table 2: $\quad$ Velocity and WSS peak values and recirculation length.

\begin{tabular}{lcc}
\hline \hline & Newtonian & Non-Newtonian \\
\hline \hline Minimum Velocity (m/s) & -0.3544 & -0.3663 \\
Maximum Velocity (m/s) & 1.262 & 1.240 \\
Recirculation length $(\mathrm{mm})$ & 24.5 & 24.5 \\
Recirculation width $(\mathrm{mm})$ & 15.7 & 13.7 \\
Maximum WSS $(\mathrm{Pa})$ & 30.9 & 22.3 \\
\hline \hline
\end{tabular}




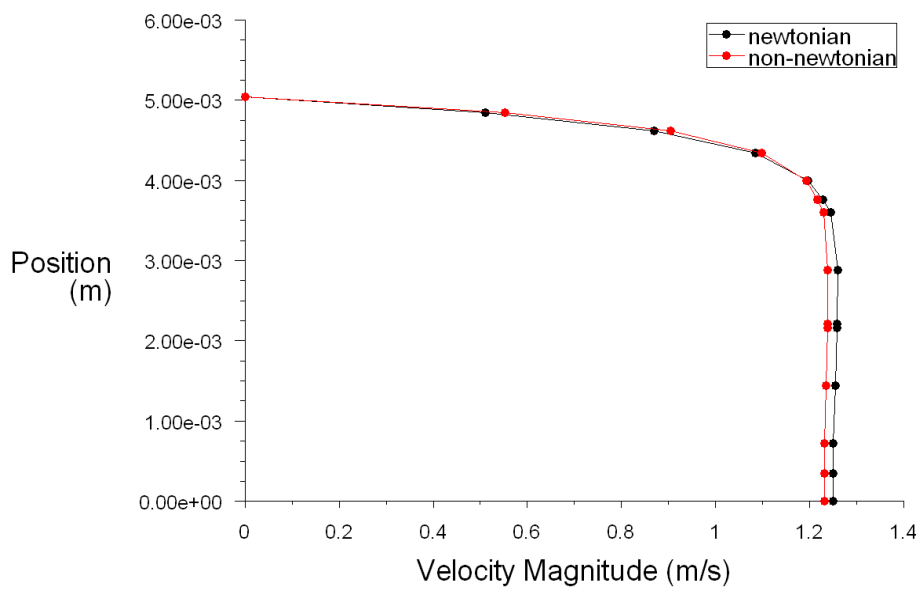

Figure 5: Velocity profile for the non-Newtonian fluid, at $x=165 \mathrm{~mm}$.

Newtonian simulations. The maximum velocity is obtained just upstream the bifurcation, but the maximum value is $1.7 \%$ lower for the non-Newtonian fluid. The minimum velocity is located downstream the bifurcation near the outer wall, $4.34 \mathrm{~mm}$ in radial direction. Although, for the non-Newtonian fluid, the minimum velocity is higher in magnitude $(3.3 \%)$, the recirculation width on the wall is smaller $(-12.5 \%)$.

Figure 6 shows the WSS values along the x direction of abdominal aorta wall, iliac outer wall and iliac inner wall, respectively.

The WSS behaviour is similar for the three walls. The higher differences are observed for the WSS maximum peak at the iliac inner wall, with a decrease of $28 \%$ for the non-Newtonian fluid. This atherosclerosis lesion factor, downstream the iliac inner wall, that is also observed clinically, is more evident with the Newtonian solution. The WSS values are always lower for the non-Newtonian fluid, which is at odds with the higher velocity gradients near the wall (Figure 5). This may be due to the variation of WSS along the radial direction, for the nonNewtonian simulation. Viscosity profile, which directly affects the WSS values, can be observed in detail in the Figure 7.

The viscosity is lower near the wall and higher at the aorta abdominal axis. As expected, near the wall, the viscosity value for the non-Newtonian fluid is lower than for the Newtonian fluid $(0.0035 \mathrm{~kg} / \mathrm{m} . \mathrm{s})$ and, consequently, WSS value is also lower.

\section{Conclusions}

A numerical CFD model to simulate the blood flow dynamics has been implemented. The flow was modelled for both a Newtonian and a shear-thinning non-Newtonian fluid. In both cases the occurrence of flow separation and the characteristics of the recirculation zone in the vicinity of iliac bifurcation have shown a similar behaviour. Similar velocity profiles are obtained, but the 

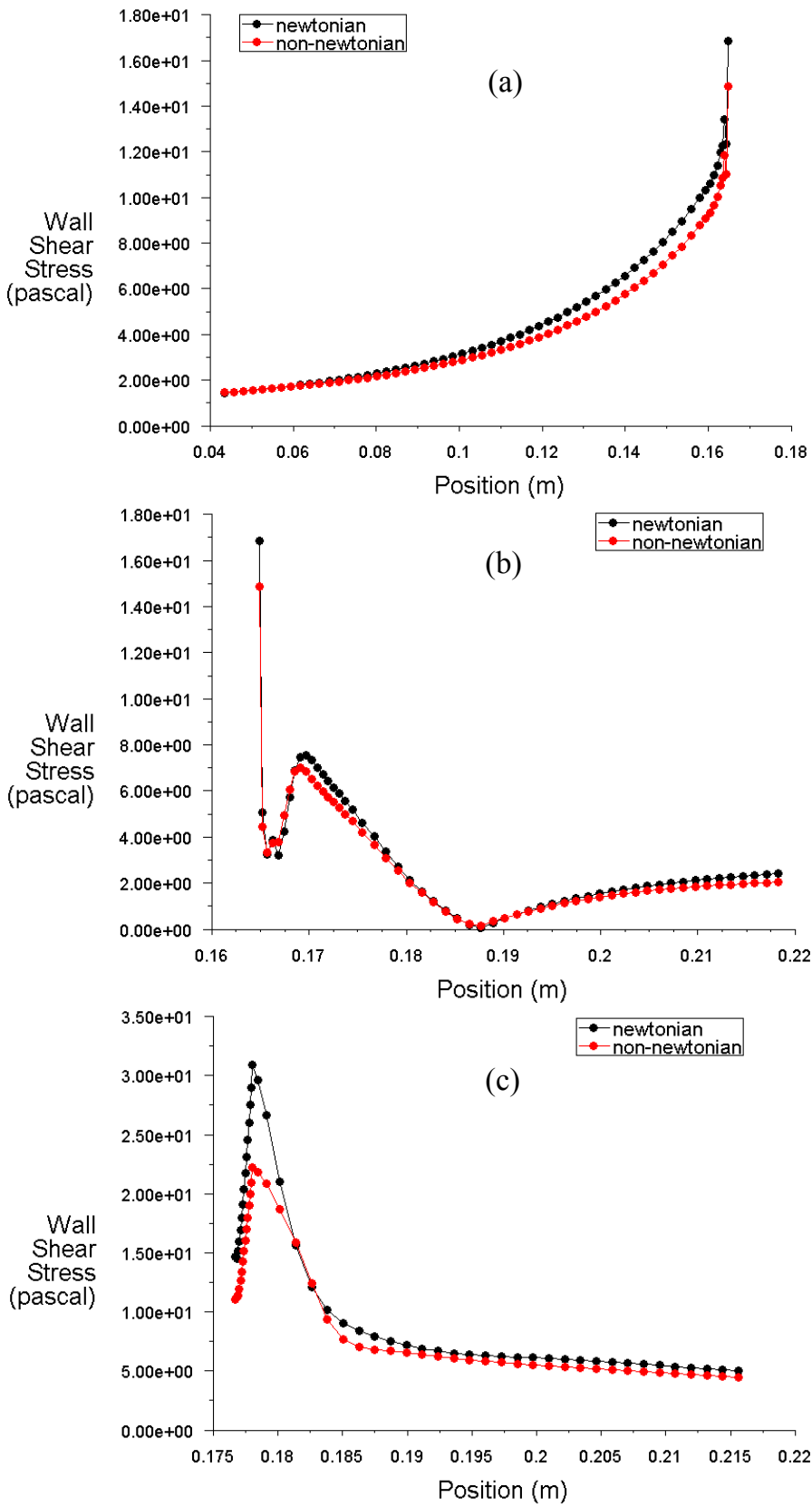

Figure 6: Wall shear stress along $\mathrm{x}$ direction, for the Newtonian and nonNewtonian simulations at: (a) abdominal aorta wall; (b) iliac outer wall; (c) iliac inner wall. 


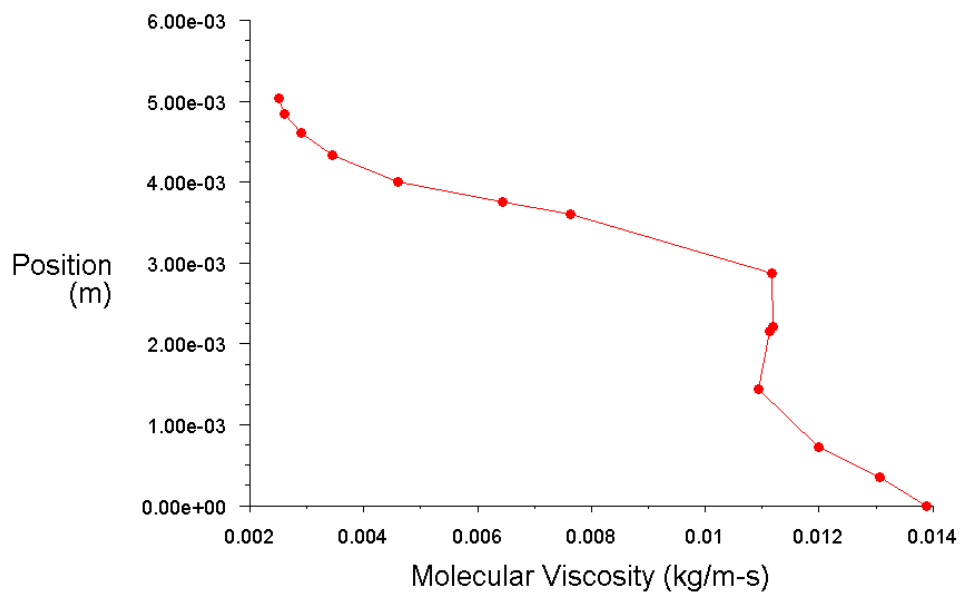

Figure 7: Molecular viscosity for the non-Newtonian fluid, just upstream from the bifurcation, at $\mathrm{x}=165 \mathrm{~mm}$.

velocity contours for a non-Newtonian fluid are flattened when compared with the Newtonian fluid. The wall shear stress values are lower for the nonNewtonian solution, which can be explained by its lower apparent viscosity in high shear regions, near the wall. Small differences were achieved with these large arteries. Both the Newtonian and the non-Newtonian model assumptions are valid for hemodynamic simulations in the abdominal aorta.

Such studies may be important tools to improve the design of stent devices or to understand drugs distribution in the circulatory system. However, further studies should consider the flow unsteadiness to analyse the wall shear stress oscillation through the cardiac cycle.

\section{Acknowledgements}

The author Filipa Carneiro gratefully acknowledges the Portuguese Foundation for Science and Technology (FCT) for the support of this work, through the grant SFRH/BD/31793/2006.

\section{References}

[1] Papaioannou, T.G., Karatzis, E.N., Vavuranakis, M., Lekakis, J.P, Stefanadis, C. (2006) Assessment of vascular wall shear stress and implications for atherosclerotic disease, International Journal of Cardiology, 113(1) 12-18.

[2] Chen, J., Lu, X-Y (2006) Numerical investigation of the non-Newtonian pulsatile blood flow in a bifurcation model with a non-planar branch, Journal of Biomechanics, 39(5) 818-832. 
[3] Owens, R. G. (2006) A new microstructure-based constitutive model for human blood, Journal of Non-Newtonian Fluid Mechanics, 140(1-3) 57-70.

[4] Johnston, B. M., Johnston, P. R., Corney S., Kilpatrick, D. (2004) NonNewtonian blood flow in human right coronary arteries: steady state simulations, Journal of Biomechanics, 37(5) 709-720.

[5] Perktold, K., Rappitsch, G. (1995) Computer simulation of local blood flow and vessel mechanics in a compliant carotid artery bifurcation model, Journal of Biomechanics, 28(7) 845-856.

[6] Gijsen, F. J. H., van de Vosse, F. N., Janssen, J. D. (1999) The influence of the non-Newtonian properties of blood on the flow in large arteries: steady flow in a carotid bifurcation model, Journal of Biomechanics, 32(6) 601608.

[7] Carneiro, F., Ribeiro, V. G., Teixeira, J. C., Teixeira, S. F. C. F. (2008) The grid scheme effect on hemodynamics numerical analysis in the iliac arteries. $8^{\text {th }}$ World Congress on Computational Mechanics, ECCOMAS 2008, June 30 - July 5, 2008, Italy (Accepted for oral presentation). 